\title{
The British Journal of Sociology
}

\section{HISTORY AND SOCIOLOGY}

\section{Volume 27, Number 3, September 1976}

This special issue of commissioned articles deals with a wide range of problems concerned with the relationship of sociology and history. Among the topics treated are the status of theory in history and sociology, the significance of the historical perspective in the social sciences, the problematic status of historical data for the sociologist, the contribution of historiography to the development of sociology, and the particular and respective contribution of history and sociology to the analysis of the social process and change.

Contributors include Peter Laslett, Guenther Roth, Gareth Stedman Jones, Immanuel Wallerstein and Edward Thompson. The issue also includes reviews of texts with special relevance to the discussion of the general theme of the issue.

Copies available from Journals Manager,

Routledge Journals,

Broadway House, Newtown Road,

Henley-on-Thames, Oxon RG9 1EN

Single issues $-£ 2.50$ each (postage included)

Annual subscription - $£ 9.00$ (postage included)

Just published:

\section{THE DIALECTIC OF CIVIL SOCIETY}

by Lawrence Krader

Series: Dialectic and Society, Nr. 2 1977. 272 pages

Price: Dutch florins 55,00 cloth

In this book the history and dialectic of civil society are explored in outline. Civil society is the society of opposed social classes and the state. Its history is the history of the transition from social bondage to the formal freedom of labor in society. The history of modern society, which is dominated by production of capital, requires such a schema that arches over the histories of particular societies that make it up, and is at once freed from the bondage to categories of European history. For these reasons, negative and positive, the central place is given to the history of civil society, both in reference to its base, the Asiatic, servile and modern modes of production, and to the origin of state. Bourgeois society is thus seen to be but a moment of the history of civil society, whose theory Ibn Khaldun and Hegel foreshadowed. The book then has an unitary burden, whose several aspects are examined in related essays. Most of these appear for the first time in print.

contents: Preface; Introduction; On the dialectic of civil society; Agrarian communism; Early history of the labour theory of value; On value; On the dialectic of anthropology.

Available through your bookshop or directly from the publishers VAN GORCUM - P.O. BOX 43 - ASSEN, The Netherlands 


\section{A N N A L E S \\ ÉCONOMIES - SOCIETES - CIVILISATIONS}

Revue bimestrielle, fondée en 1929 par

LUCIEN FEBVRE et MARC BLOCH

Comite de Direction:

FERNAND BRAUDEL, MARC FERRO, GEORGES FRIEDMANN, JACQUES LE GOFF, EMMANUEL LE ROY LADURIE, CHARLES MORAZE

Secrétaires du Comité: PAUL LeUILliot, MARIANNE MAHN-LOT, ANDRE bURGUIËRE Secrétaire de la Rédaction: JACQUES REVEL

31e ANNÉE - No 3 - MAI-JUIN 1976

\section{Mythes et religions}

Stefano Varese et Moisés Gamarra, Deux versions cosmogoniques campa: esquisse analytique

Michel Zink, Moulin mystique. A propos d'un chapiteau de Vézelay: figures allégoriques dans la prédication et dans l'iconographie romanes

M. H. Froeschlé-Chopard, Univers sacré et iconographie au XVIIIe siècle: églises et chapelles des diocèses de Vence et de Grasse

Le christianisme et l'Eglise (Comptes rendus, par Jeanine Brun-Rovet, Solange Deyon, Claude Langlois, Claude Lepelley, Jean-Marie Martin, Jean-Claude Schmitt, Jacques Solé, Huguette Taviani, Bernard Vogler)

\section{Le récit et l'histoire}

Denise Maldidier et Régine Robin, Du spectacle au meurtre de l'événement: reportages, commentaires et éditoriaux de presse à propos de Charléty (Mai 1968)

Linguistique et sémiologie (Comptes rendus, par Pierre-Yves Badel, Bernard Cerquiglini, Marie-Renée Guyard)

\section{Recherches en cours}

Gérard Sivéry, Les profits de l'éleveur et du cultivateur dans le Hainaut à la fin du Moyen Age

François Sigaut, Pour une cartographie des assolements en France au début du XIXe siècle

Tihomir J. Markovitch, La croissance industrielle sous l'Ancien Régime

Summaries of articles

Rédaction: 54, Boulevard Raspail, 75006-Paris

Administration: Librairie Armand Colin, 103, Boulevard Saint-Michel, 75005-Paris

Comptes chèques postaux: Paris, No 21 335-25

Abonnements: France et Pays de la Communauté: $110 \mathrm{~F}$ (Etudiants: $95 \mathrm{~F}$ ) - Etranger:

$140 \mathrm{~F}$ - Le numéro: $23 \mathrm{~F}$ - Numéros spéciaux : $46 \mathrm{~F}$ 


\section{A N N A L E S \\ ECONOMIES - SOCIETES - CIVILISATIONS}

Revue bimestrielle, fondée en 1929 par

LUCIEN FEBVRE et MARC BLOCH

\section{Comité de Direction:}

FERNAND BRAUDEL, MARC FERRO, GEORGES FRIEDMANN, JACQUES LE GOFF, EMMANUEL LE ROY LADURIE, CHARLES MORAZÉ

Secrétaires du Comité: PAUL LEUILLIOT, MARIANNE MAHN-LOT, ANDRÉ BURGUIÈRE Secrétaire de la Rédaction: JACQUES REVEL

31e ANNÉE - No 4 - JUILLET-AOOT 1976

\section{ANTHROPOLOGIE DE LA FRANCE}

Jean Bernard et Jacques Ruffié, Hématologie et culture. Le peuplement de l'Europe de l'Ouest

P.-A. Gloor et Jacques Houdaille, La couleur des yeux à l'époque du Ier Empire. Quelques commentaires sur l'évolution de ce caractère durant 238 ans

Michel Demonet, Paul Dumont, Emmanuel Le Roy Ladurie, Anthropologie de la jeunesse masculine en France au niveau d'une cartographie cantonale (18191830)

Fernand Boulard, Matériaux pour l'histoire religieuse du peuple français. Aspects de la pratique religieuse en France, 1802-1939: l'exemple des Pays de Loire

Marie-Noëlle Bourguet, Race et folklore. L'image officielle de la France en 1800 Yvonne Knibiehler, Les médecins et la "nature féminine" au temps du Code civil

A travers la France (Comptes rendus, par Maurice Agulhon, Alain Collomp, Daniel Dessert, Daniel Fabre, Jacques Lafon, Laurent Lévi-Strauss, Pierre Ponsot, Pierre Sorlin, Pierre Souyri)

Summaries of articles

Rédaction: 54, Boulevard Raspail, 75006-Paris

Administration: Librairie Armand Colin, 103, Boulevard Saint-Michel, 75005-Paris

Comptes chèques postaux: Paris, No 21 335-25

Abonnements: France et Pays de la Communauté: $110 \mathrm{~F}$ (Etudiants: $95 \mathrm{~F}$ ) - Etranger:

$140 \mathrm{~F}$ - Le numéro: $23 \mathrm{~F}$ - Numéros spéciaux: $46 \mathrm{~F}$ 


\section{AVRIL-JUIN 1976 \\ Numéro 95

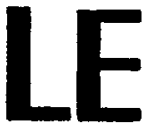 \\ $\mathrm{MO}$

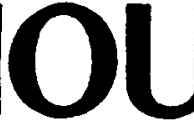 \\ JEMEN
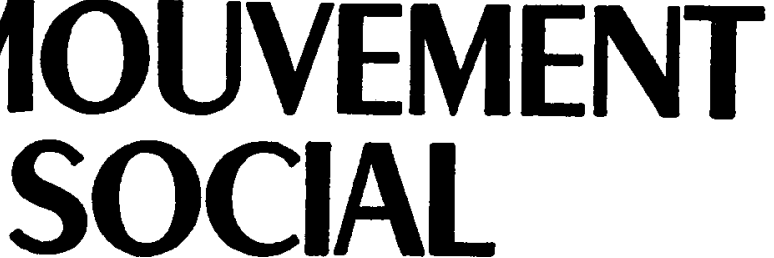

Revue trimestrielle publiée avec le concours du Centre National de la Recherche Scientifique et avec la collaboration du Centre d'Histoire du Syndicalisme de l'Université de Paris I (Panthéon-Sorbonne)

\section{SOMMAIRE}

\section{ASPECTS DU SOCIALISME ALLEMAND sous la direction de Jacques Droz}

Historiographie d'un siècle de social-démocratie allemande, par Jacques Droz

Du socialisme vrai au sionisme socialiste. L'itinéraire d'un prophète: Moïse Hess, par Alain Boyer

Le socialisme scientifique de Ferdinand Lassalle, par Sonia DayanHerzbrun

Intégration négative et attentisme révolutionnaire, par Dieter Groh La reconstruction du SPD après la Deuxième Guerre mondiale, par Françoise Foret

Réflexions sur un livre: Rosa Luxemburg: mythe et réalité, par Emile Bottigelli

Informations scientifiques

Résumés en français et en anglais

\section{LES EDITIONS OUVRIERES}

\section{2, avenue de la Soeur-Rosalie - 75621 Paris - Cédex 13}

Abonnement annuel: France: 60 F., Etranger: 70 F. C.C.P. Paris 1360-14 Le numéro: France: 18 F., Etranger: $20 \mathrm{~F}$. 


\section{JUILLET-SEPTEMBRE 1976 \\ Numéro 96 \\ LE

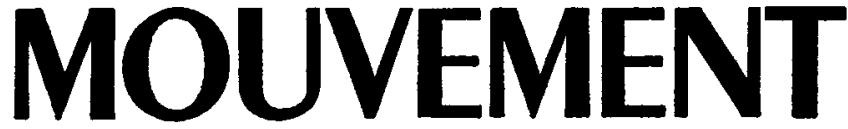 \\ SOCAL}

Revue trimestrielle publiée avec le concours du Centre National de la Recherche Scientifique et avec la collaboration du Centre d'Histoire du Syndicalisme de I'Université de Paris I (Panthéon-Sorbonne)

\section{SOMMAIRE}

Les grèves du Front populaire de mai et juin 1936. Une nouvelle analyse fondée sur l'étude de ces grèves dans le bassin houiller du Nord et du Pas-de-Calais, par Raymond Hainsworth

Juillet à septembre 1936 à Cadix (Espagne) : les travailleurs face aux militaires, par Maryse Esterlé

Recherches sur la morphologie du corps universitaire littéraire sous la Troisième République, par Victor Karady

Enseignement secondaire et idéologie élitiste entre 1880 et 1914, par Suzanne Citron

L'enseignement secondaire des jeunes filles (1867-1924), par Françoise Mayeur Sur l'histoire du mouvement trotskyste en Europe de 1930 à 1952, par Michel Dreyfus

\section{Notes de lecture}

Lassalle, par S. Na'aman (J. Droz). Les Marxistes et la question nationale, 1848-1914, par G. Haupt, M. Lowy, Cl. Weill (R. Gallissot). Eduard Bernsteins Briefwechsel mit Friedrich Engels, par H. Hirsch (G. Badia). R. Luxemburg, Lettres à Léon Jogichès, t. 1: 1894-1899; t. 2: 1900-1914 (J. Girault). Cinquante ans de rapports entre patrons et ouvriers en Allemagne, $t$. II, depuis 1945, par P. Waline (Cl. Weill).

Courrier des lecteurs

Résumés en français et en anglais

\section{LES EDITIONS OUVRIERES}

12, avenue de la Sœur-Rosalie - 75621 Paris - Cédex 13

Abonnement annuel: France: 60 F., Etranger: 70 F. C.C.P. Paris 1360-14 Le numéro: France: $18 \mathrm{~F}$, Etranger: $20 \mathrm{~F}$. 


\section{WISSENSCHAFTLCHE PAPERBACKS}

\section{Sozial- und Wirtschaftsgeschichte}

Hiragugebun won HANS POH.

Je Band ca. 200 Seiten, DM 18,一, bei Reihenbezug DM 16,-

\section{Studien zur Wirtschaftsgeschichte Lateinamerikas}

\section{Von Hans Pohl}

1976. VIII, 287 Seiten. ISBN 3-515-02388-7

Der Band faßt die Aufsätze des Autors aus einem Jahrzehnt wirtschaftshistor ischer Lateinamerikaforschung zusammen, die um die drei großen Themenbereiche Handels- und Verkehrsgeschichte, Gewerbe- und Industriegeschichte sowie Agrargeschichte kreisen.

\section{Die deutsche Wirtschaft in der Zwischenkriegszeit}

\section{Von Dietmar Petzina}

1976. VIII, 205 Seiten. ISBN 3-515-02173-6

In der vorliegenden Studie werden die besonderen Probleme der wirtschaftlichen und sozialen Entwicklung Deutschlands im Zeitraum zwischen den Weltkriegen dargestellt. Der Text ist durch einen umfangreichen Tabellenteil und eine Auswahlbibliographie ergänzt, um dem Leser Anregungen für ein vertieftes Studium zu vermitteln.

Bereits lieferbar:

I Ernst Klein: Geschichte der deutschen Landwirtschaft im Industriezeitalter 1973. VIII, 192 Seiten. ISBN 3-515-01190-0

2 Hans Jaeger: Geschichte der amerikanischen Wirtschaft im 20. Jahrhundert 1973. VIII, 186 Seiten. ISBN 3-515-01188-9

3 Fritz Blaich: Die Epoche des Merkantilismus 1974. VIII, 203 Seiten. ISBN 5-515-01197-8

4 Harald Winkel: Die Wirtschaft im geteilten Deutschland r945-r970 1974. XIV, 217 Seiten m. 81 Tab. u. 6 Abb. ISBN 3-515-01799-2

5 Albin Gladen: Geschichte der Sozialpolitik in Deutschland Eine Analyse ihrer Bedingungen, Formen, Zielsetzungen und Auswirkungen 1974. X, 201 Seiten. ISBN 3-515-01807-7

6 Ernst Klein: Geschichte der öffentlichen Finanzen in Deutschland (I500-r870). 1974. VIII, 145 Seiten. ISBN 3-515-01880-8

7 Winfried Baumgart: Der Imperialismus Idee und Wirklichkeit der englischen und französischen Kolonialexpansion 1880-1914. 1975. VIII, 179 Seiten, 1 Karte. ISBN 3-515-01969-3

8 Géza Alföldy: Römische Sozialgeschichte 1975. X, 239 Seiten. ISBN 3-515-02045-4

9 Thomas Pekáry: Die Wirtschaft der griechisch-römischen Antike 1976. X, 134 Seiten. ISBN 3-515-02154-X

FRANZ STEINER VERLAG GMBH • D-6200 WIESBADEN P.0.B. 5529 